\title{
An abnormal bony union between leg bones
}

\author{
Umesan KG
}

\begin{abstract}
Introduction: An osteology specimen of post traumatic proximal tibiofibular synostosis is discussed along with photographs of the specimen and its $X$-ray and computed tomography scan. Case Report: An osteology specimen of tibia and fibula united in their proximal shafts was studied. The bony bridge between the leg bones was associated with a callus in the tibia. Computed tomography scan showed full grown cortex and marrow cavity inside the synostosis. The inferior tibiofibular joint was disrupted. Conclusion: An osteology specimen of post traumatic proximal tibiofibular synostosis is discussed. Similar occurance in life can lead to peroneal neuropathy and persistent ankle pain.
\end{abstract}

Keywords: Proximal, Tibiofibular, Synostosis

$* * * * * * * * *$

Umesan KG. An abnormal bony union between leg bones. International Journal of Case Reports and Images 2013;4(6):334-336.

$* * * * * * * * *$

doi:10.5348/ijcri-2013-06-325-CR-10

Umesan KG

Affiliations: Assistant Professor, Govt. T.D. Medical College, Alappuzha, Kerala, India

Corresponding Author: Dr. Umesan KG, T.C.27/532, Raj Bhavan, Thampuranmukku Vanchiyoor P.O. Trivandrum, Kerala, India 695035; Email: umesankg@yahoo.com

Received: 28 October 2012

Accepted: 22 February 2013

Published: 01 June 2013

\section{INTRODUCTION}

Eventhough distal tibiofibular synostoses are common occurrences in orthopedics, a proximal tibio fibular synostosis is not much heard of. An osteology specimen showing a post-traumatic proximal tibiofibular synostosis is discussed here.

\section{CASE REPORT}

Description of specimen: An osteology specimen of tibia and fibula with proximal tibiofibular synostosis in the upper third of the unit is described. The bones belong to left side. The tibia is $31.4 \mathrm{~cm}$ long and the fibula is $31.6 \mathrm{~cm}$ long. The proximal tibiofibular joint space is normally maintained. The posterior and inferior margins of the superior fibular articular surface show osteophytic activity. The tibia is deformed in the proximal $1 / 6$. There is evidence of partially remodeled callus at the junction of the upper $1 / 6$ of the tibia with the rest of the bone.

The proximal part of the synostosis is displaced slightly medially (Figure 1). The callus causes a fusiform bulge around the proximal tibial shaft more to the medial and posterior aspects. The bone in this area shows multiple vascular foramina and trabeculae. Extending from the lateral aspect of this area, is a bony bridge to the fibula measuring $8 \mathrm{~mm}$ horizontally and 12 $\mathrm{mm}$ vertically and $2.5 \mathrm{~mm}$ antero posteriorly meeting the latter along the interosseous border and merging with it. The rest of the shafts of the tibia and fibula do not show any abnormality.

The tibia shows an apparent shortening of about $5 \mathrm{~mm}$ as a result of the fracture reunion. The synostotic area shows smooth surfaced bone. The inferior tibio fibular articulation appears disrupted and the joint space is $8 \mathrm{~mm}$.

CT scan shows osseous continuity between the bones with cancellous bone within the synostosis. The red arrows in Figure 2 indicate the marrow cavity with trabecular bone inside. 


\section{DISCUSSION}

Union between adjacent bones or parts of a single bone made of osseous material, such as ossified connecting cartilage or fibrous tissue constitutes a synostosis. Etiologically, it may be congenital or acquired. In the latter group are the post traumatic, iatrogenic and the therapeutic types. A tibiofibular synostosis may be proximal or distal in position.

The congenital tibio fibular synostosis is due to persistence of an embryonal development stage of incompletely separated of the cartilage common to tibia and fibula during development of these bones $[1,2]$. If a synostosis occuring before closure of the epiphysis, there may be associated deformities like distally placed proximal tibiofibular joint, leg length discrepancy, bowing of the fibula, or valgus deformity of the knee.

The first reported case of proximal tibiofibular synostosis was by Rahm in 1924. His 43-year-old female patient had a unilateral genu valgum for a corrective osteotomy was done. Synostoses due to multiple hereditary exostoses may present with intermittent peroneal neuropathy. Namihira tatsukuni reported a case of an eight-year-old girl with X-rays showing a proximal tibio fibular synostosis. She was reported to have left heel in more valgus, the longitudinal arch of the left foot lower and internal rotation of the patella as associated anomalies [3].

Post-traumatic distal tibiofibular synostosis may follow tibial and ankle fractures but midshaft, or proximal tibiofibular synostoses are also probable. Soft tissue damage with bleeding across the interosseous membrane may end up in new bone formation. As the tibia and fibula are bound by three joints, they act as a single unit and any change of normal anatomy at one end can affect the function at the other end.

Iatrogenic proximal tibiofibular synostosis may follow nailing and osteotomy for reduction of tibial fractures. The etiology may be injury to the soft tissues, hemorrhage or subperiosteal dissection across the interosseous membrane leading to new-bone formation [4]. The postoperative bridge-callus arising from instability of the osteotomy-area forms the tibiofibular synostosis.

The proximal tibiofibular joint helps to reduce the ankle torsional stresses, minimises weight-bearing tensile stresses. Frick observed that a synostosis may interfere with the normal movement between the tibia and fibula during weight-bearing leading to persistent ankle pain aggravated during the push-off phase of walking. This happens from failure of the normal downward and lateral motion of the fibula while in maximum weight bearing and stress. It may even cause a stress fracture of the fibula.

S.L. Mubarak found a reversal in the normal growth pattern of distal migration of the fibula relative to the tibia causing a decreased distance between the proximal physes of the tibia and fibula as well as proximal migration of the distal fibular physis relative to the distal part of the tibia [5]. The synostosis prevents the normal distal movement of the fibula relative to the tibia

Figure 2: Computed tomography scan showing cross sectional appearance of the proximal tibiofibular synostosis. Upper left-Normal. Others-varying parts of the synostosis. 
producing shortening of the lateral malleolus and ankle valgus as well as prominence of the fibular head at the knee.

A synostosis can be left alone if the movements at the ankle joint are normal. If symptomatic, excision of the synostosis or fibular osteotomy can be done after the synostosis has matured. Osteotomy at the junction of the middle and distal thirds of the fibula helps to reduce the mechanical stress on the proximal tibiofibular joint coming from the ankle joint. Peroneal nerve branches to the extensor hallucis longus can be injured during this [6].

Therapeutic tibiofibular synostosis in below-knee amputation, described by von Ertl in 1949, offers theoretical benefits for prosthetic end [7].

\section{CONCLUSION}

An osteology specimen of Post traumatic Proximal Tibio Fibular Synostosis is presented.

$$
* * * * * * * *
$$

\section{Acknowledgements}

Dr. Shobha Ramnarayan, Professor \& HOD, Department of Anatomy, Govt. Medical College, Trivandrum.

\section{Author Contributions}

Umesan KG - Conception and design, Acquisition of data, Analysis and interpretation of data, Drafting the article, Critical revision of the article, Final approval of the version to be published

\section{Guarantor}

The corresponding author is the guarantor of submission.

\section{Conflict of Interest}

Authors declare no conflict of interest.

\section{Copyright}

(C) Umesan KG et al. 2013; This article is distributed under the terms of Creative Commons Attribution 3.0 License which permits unrestricted use, distribution and reproduction in any means provided the original authors and original publisher are properly credited. (Please see www.ijcasereportsandimages.com/copyright-policy.php for more information.)

\section{REFERENCES}

1. von Törne O. [Tibiofibular synostoses. Z Orthop Ihre Grenzgeb 1977 Jun;115(3):372-5.

2. Nishimura T, Nii E, Urawa M, Nishiyama M, Taki S, Uchida A. Proximal tibiofibular synostosis with 49,XXXXY syndrome, a rare congenital bone anomaly. J Orthop Sci 2008 Jul;13(4):390-5.

3. Namihira tatsukuni. A Case of Proximal Tibiofibular Synostosis. Orthopedics \& Traumatology 2000;49(2):502-5.

4. Mandal S, Coleman N. Proximal tibiofibular synostosis following tibial nailing: a case report and review of literature. Injury Extra 2005;36(10):418-20.

5. Frick SL, Shoemaker S, Mubarak SJ. Altered fibular growth patterns after tibiofibular synostosis in children. J Bone Joint Surg Am 2001 Feb;83$\mathrm{A}(2): 247-54$.

6. Gamble JG. Proximal tibiofibular synostosis. J Pediatr Orthop 1984;4(2):243-5.

7. Keeling JJ, Schon LC. Tibiofibular Bridge Synostosis in Below-Knee Amputation. Techniques in Foot \& Ankle Surgery 2007 September;6(3):156-61.
Access full text article on other devices

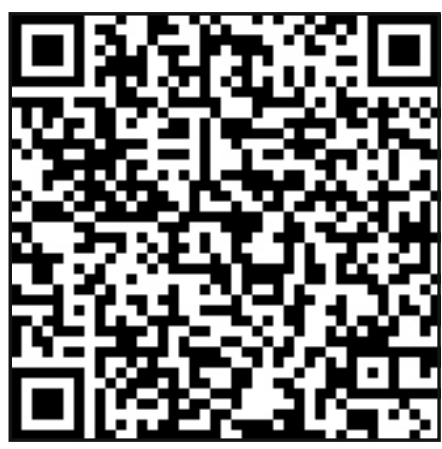

Access PDF of article on other devices

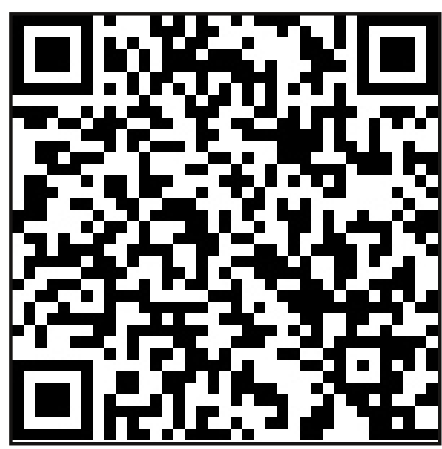

